\section{Effect of drive level on habit strength in a discrimination task}

JHROME: S. COHLI and (iABOR A. TElLGDS. Cintersity of Windsor. l'indsur 11. Ont. Canada

Effects of drive level on habit strength of a brightness discrimination were determined for male albino rats. Habit strength was assessed by measuring response strength of the old stimulis, compared with a newer trained redumdant positive cue. Only in one trpe of test trial was a drive effect on habit strength found. Moderately nater-deprived Ss (MOD). regardless of drive level during redundant (twe training or testing, preferred the redundant cue to the positive stimulus. Highl!. deprived $S_{S}$ (HI) showed no prifirence. but responded more to the . kice positive stimulus than did MOD Ss. These results support the hypothesis that habit strength is directly related to drive level. The specific conditions under which drive level effects on learning could be most easily obsericed were discussed.

Drive level has been postulated to affect learning by having an inverse effect on span of attention (Tolman, 1948; Easterbrook, 1959). Animal studies measuring cue utilization tend to support this view. Bruner et al (1955) found that lower motivated (hunger) rats were able to utilize concomitant position cues when brightness cues had been removed from an alternation task with four choice points. Highly deprived Ss, however, were unable to perform the alternating sequence when brightness cues had been removed. Cohen et al (1969) trained rats under two drive (thirst) conditions in a discrimination task with two redundant dimensions, shape and size. Lower water-deprived Ss were found to be able to utilize both cues separately. whereas highly deprived Ss only used size cues in the discrimination task. These studies failed to counterbalance drive conditions. and, therefore, failed to show that differences in cue utilization were actual learning rather than performance phenomena.

Eisman et al (1956) counterbalanced three levels of drive (hunger) for rats run in a brightness discrimination task. The lack of differences in original learning and reversal learning between the three drive levels signified for these investigators that drive level does not affect amount of habit strength.

The present study was designed to measure the differences in habit strength due to drive level (thirst) in a simple discrimination task and to assess the transfer of such differences over later drive-level changes. Ss were trained on a simple brightness discrimination task under conditions of high water deprivation (HI group) or moderate water deprivation (MOD group). After reaching acquisition criterion, a redundant stimulus was placed on the positive stimulus door and a series of discrimination trials was continued. To test for the strength of the original discrimination. the redundant cue was placed in the apparatus such that $S$ had to choose between it and the positive stimulus that had been presented during the original acquisition trials. It was predicted that under these test conditions. HI Ss should respond more to the originally learned positive stimulus than should MOD Ss, and that $\mathrm{HI}$ Si should prefer the originally learned positive stimulus to the redundant stimulus. while MOD $\mathrm{S} s$ should show no such preference. These predictions were based on the assumption that during original discrimination MOD $\mathrm{Ss}$ would attend to more cues than would HI Ss. Therefore, any particular stimulus should not have as much strength in eliciting a response for MOD Ss as for $\mathrm{HI}$ Ss during training or testing conditions. If these effects could be demonstrated over drive level changes from the level under the original discrimination training, then habit strength for the earlier learned task could be considered greater for HI than for MOD Ss.

\section{SLIBJECTS}

Thirty male albino rats from the breeding colonies of the University of Windsor and Woodlyn Farms, Guelph, Ontario, approximately 90 days old at time of pretraining. were used. At the time of discrimination training, half the $S s$ were assigned randomly to either a high water-deprivation schedule (HI) or to a moderate water-deprivation schedule (MOD). Each deprivation group was divided randomly into three subgroups: HI/HI or MOD/MOD experimental, HI/MOD or MOD/HI experimental, and HI or MOD control subgroups. The HI/MOD or $\mathrm{MOD} / \mathrm{HI}$ designates drive-level change from discrimination acquisition drive level to that for redundant cue and testing phases. Three MOD and two HI Ss were discarded due to failure to meet running criterion. They were replaced by extra Ss in order to maintain equal subgroup $\mathrm{Ns}_{\mathrm{s}}$ for statistical analysis.
APPARATUS

A discrimination box as used in the Cohen et al (1969) study was employed in this study.

\section{PROCEDURE \\ Pretraining}

Essentially the same pretraining procedures as used in the Cohen et al (1969) study were employed. The only changes made were to lengthen sucrose solution taming and free exploration of the apparatus to 5 days each. In training $S$ to open the stimulus doors to secure reinforcement, 4 days of one 4-min trial and 12 spaced trials on the 5 th day were given to each $\mathrm{S}$. Preference to either door was discouraged by locking the preferred door after six trials for the remaining trials. Discrimination Training

Two deprivation groups of 15 Ss each were maintained on either a high deprivation schedule (HI group) of $0.5 \mathrm{~h}$ water per $24 \mathrm{~h}$ or were continued on the previous moderate schedule (MOD group) of $6 \mathrm{~h}$ ad lib drinking per $24 \mathrm{~h}$. In the discrimination box, the gray door was the positive stimulus and always led to the sucrose solution reinforcement. The black door was the negative stimulus and was always locked. This phase will be designated as a $\mathrm{G}+$ vs $\mathrm{B}$ - discrimination throughout the text.

In all phases, $S$ was given 12 spaced trials per day. A trial consisted of the number of runs made until S chose the correct door. $S$ was replaced in the startbox when it had made an error. The series of random position sequences described by Fellows (1967) was used only between trials and not between runs on any one trial. An error was considered to have been made only when $S$ pushed on or touched the incorrect door (B-) with its nose. Since each door when touched could budge about 0.25 in., observing mistakes was not difficult. A correct response consistuted nunning to the gray door $(\mathrm{G}+)$ or switching over to it after the incorrect $\mathrm{B}$ - stimulus had been approached but not touched. $S$ reached discrimination acquisition when it made two or less errors within 24 consecutive trials.

Redundant Cue Presentation (Gwc+ vs B-)

After reaching acquisition criterion, $\mathrm{S}$ was assigned randomly to one of three subgroups. Two of these groups consisted of Ss that received discrimination trials with a redundant stimulus. These were considered experimental groups. The third group of $\mathrm{Ss}$ was immediately assigned to the test phase and was considered in a control group. Within each drive-level group, therefore, there were three subgroups of five Ss each. The control groups were maintained on their same drive 
level as during discrimination learning. They are designated HI control and MOD control groups. Of the two experimental subgroups within each drive level, one was maintained on its previous drive level and given the redundant cue presentation trials. These $\mathrm{Ss}$ are designated $\mathrm{HI} / \mathrm{HI}$ experimental and MOD/MOD experimental. The other experimental group was placed on the opposite deprivation schedule, i.e., HI Ss switched to the moderate deprivation level and MOD Ss switched to the high deprivation schedule. Such Ss are designated HI/MOD and MOD/HI experimental. The first syllable designates the schedule during the discrimination, while the second designates the drive-level change. These Ss were retumed to their home cages for 3 days and were presented the redundant cue on the 4th day.

The redundant cue was a white 2-in.-diam circle attached to the gray door on its midline. This redundant positive stimulus was placed so that its bottom edge was $1 \mathrm{in.}$ from the floor of the apparatus. The stimulus was constructed from white poster board, $1 \mathrm{~mm}$ in thickness. Twelve spaced corrected trials were given with the simultaneous presentation of the gray door with the white circle and the black door. The gray door stimulus complex was again positive and the black door negative. These series of stimuli presentations are designated $\mathrm{Gwc}+\mathrm{B}-$.

The presentation of these trials after the drive level change allowed $S$ to modify its behavior due to drive changes. In this way, the study was designed to maximize for any preformance effects due to drive level change. The degree to which behavior of the HI/MOD and MOD/HI Ss resembled $\mathrm{HI} / \mathrm{HI}$ and MOD/MOD experimental Ss indicated the effect of the original drive level on discrimination learning.

\section{Testing}

This phase was designed to test the strength of the original positive stimulus $(G+)$ against the new redundant stimulus (wct) in eliciting approach for reinforcement. After the redundant cue training trials, all experimental Ss were presented 72 discrimination trials (12 spaced corrected trials per day). Every fourth trial was a test trial, while the rest of the trials were the same as in the previous phase, i.e., Gwct vs B-. During a test trial, both stimulus doors were unlocked and $S$ could receive reinforcement by entering through either one. This procedure was also used by Sutherland \& Holgate (1966) and prevented the test preference trials from becoming specific discrimination learning trials for Ss.
Three types of test-trial stimulus pairs were used:

Test Pair a: Gray door with white cirvle vs gray door $-\mathrm{Gwc}$ vs $\mathrm{G}$.

Test Pair b: Black door with white circle vs black door-Bwc vs B.

Tèst Pair c: Black door with white circle vs gray door-Bwc vs $\mathrm{G}$.

Test Pairs a and $c$ were more appropriate pairs in testing the strength of the formerly positive stimulus $G$ against wc redundant stimulus for approach behavior. Test Pair $b$, on the other hand, was purely a test to see if the white circle, in relation to the negative black door, would also affect differentially choices between drive-level groups. Test-pair schedules for the 6 testing days were as follows for each $S$ : $a b c, c a b$, bca, cab, abc, bca. Number of times $S$ chose the door without the redundant cue was recorded.

Control group Ss received a similar testing phase. They had not been presented with the $\mathrm{Gwc}+$ vs $\mathrm{B}-$ presentation trials. During the testing phase, all nontesting trials were of the same type as during the discrimination acquisition phase, $\mathrm{G}+\mathrm{vs} \mathrm{B}-$ These Ss provided controls for novelty effect differences between drive levels. Previous studies (Chapman \& Levy, 1957; Cohen \& Stettner, 1968) have demonstrated that lower deprived Ss tend to explore novel stimuli more than highly deprived Ss. In the present study, therefore, any differences in stimulus preference between MOD and $\mathrm{HI}$ experimental subgroups might be considered as an indication of different exploratory tendencies toward a novel, i.e., white circle, stimulus. It was possible, therefore, that the redundant cue as presented in $\mathrm{Gwc}+\mathrm{vs} \mathrm{B}$ - presentations was really never attended to in the first place by $S$ and, therefore, may never have acquired positive approach characteristics. The degree of difference between control and experimental subgroups in the testing phase would reflect the extent to which drive level exploratory differences contributed to differential responding to test stimuli by experimental subgroups.

If $S$, during any phase of the study refused to enter the goal area or refused to drink within 10 min on 2 successive days, it was discarded and its data not used.

\section{RESULTS}

No significant differences between drive levels were found for mean number of trial blocks (MOD-8, HI-10: $\mathrm{t}=1.60, \mathrm{df}=28$ ) or for mean number of errors (MOD-46, HI.42: $t=0.94)$ during discrimination learning.

It must be noted that not all experimental Ss received the redundant cue presentation phase at the same time. $\mathrm{HI} / \mathrm{HI}$ and MOD/MOD were presented this phase the day following brightness discrimination, while HI/MOD and MOD/HI Ss rested 3 days before redundant cue presentations. The amount of total errors for each drive subgroup was 2, 4, 1 , 1, respectively. A Kruskal-Wallis one-way analysis of variance revealed a lack of significant differences between these groups $(\mathrm{H}=2.78)$. Thus, the difference in time of redundant cue presentation was considered an insignificant factor in this study.

A multifactor, Drive Level by Drive Manipulation by Test Pair series (repeated) analysis of variance was carried out on number of responses made to the stimulus door without the redundant stimulus during the testing phase. Significant main effects beyond the .01 critical level were found for original drive level $(F=6.07$, $\mathrm{df}=1 / 24)$, test series $(\mathrm{F}=31.50$, $\mathrm{df}=2 / 48)$ and an interaction of Drive by Test Pair series $(F=5.34 . \mathrm{d} f=2 / 48)$. Drive manipulation main effects and interactions of drive manipulation with drive level and/or test series were insignificant.

Individual comparisons (Newman-Keuls) for responses to the door without the redundant cue in each type of test pair presentation series between the different drive subgroups were carried out (see Table 1).

The significance of the main effects for type of test pair presentations seemed due to more responses made by all subgroups to $B$ door in Test Pair $b$ than to the $G$ doors in either Test Pairs a or c. All HI Ss showed differences beyond the .05 level of

Table 1

Mean Number of Responses and Standard Deviations (in Parentheses) to G Stimulus Door in Test Pairs a and $c$ and to $B$ Stimulus Door in Test Pair b

\begin{tabular}{|c|c|c|c|}
\hline \multirow[b]{2}{*}{ Drive Subgroup } & \multicolumn{3}{|c|}{ Test Pair } \\
\hline & a & $\mathrm{b}$ & c \\
\hline \multicolumn{4}{|l|}{ Experimental } \\
\hline HI & $2.4(2.3)$ & $0.4(0.3)$ & $3.6(0.8)$ \\
\hline MOD & $1.4(2.8)$ & $0.2(0.2)$ & $1.4(1.3)$ \\
\hline $\mathrm{HI} / \mathrm{MOD}$ & $1.8(1.2)$ & $0.2(0.2)$ & $2.6(2.8)$ \\
\hline $\mathrm{MOD} / \mathrm{HI}$ & $2.6(1.8)$ & $0.4(0.3)$ & $1.6(0.3)$ \\
\hline \multicolumn{4}{|l|}{ Control } \\
\hline $\mathrm{HI}$ & $2.6(0.8)$ & $1.0(5.0)$ & $3.6(0.8)$ \\
\hline MOD & $2.6+2.31$ & $0.2(0.2)$ & $2.2(2.2)$ \\
\hline
\end{tabular}


significance. The differences seen for MOD Si were only significant for MOD control Ss $(p<.05)$ and MOD/HI Ss (only Test Pairs $b>a, p<.051$. In HI Ss only. a trend was seen for more responses to be made to $G$ door in Test Pair i than a. Significance for these differences was found only in HI/MOD Ss $(\mathrm{p}<.01)$.

The significant interaction of Drive by Test Pair was accounted for by the simple effects of drive upon Test Pair c. No significant differences in responding to the plain door in either Test Pairs a ( $G$ door) or b (B dom) were found betwen various drive level subgroups. In Test Pair 2 . original predictions concerning drive level differences were found. $\mathrm{Hl} / \mathrm{Hl}$ and HI/MOD Ss made significantly more responses to the $G$ stimulus door than MOD/MOD or MOD/HI Ss, respectively $(\mathrm{p}<.01)$. Since no significant differences were found between $\mathrm{HI} / \mathrm{HI}$ and $\mathrm{HI} / \mathrm{MOD}$ or between MOD/MOD and MOD/HI Ss, drive level during the first discrimination learning phase. rather than during redundant cue presentation or the testing phases, determined the results during the latter phase. Although $\mathrm{HI}$ control Ss appeared to make more responses than MOD control Ss, this difference was not significant. Therefore. the differences found between the experimental subgroups appear more a reflection of habit strength differences rather than differences in exploratory tendencies.

A series of chi-square tests, comparing the number of responses to each stimulus for each test pair for each drive level subgroup, was used to assess stimulus preference. The number of Ss showing no preference, or a preference for either stimulus, i.e.. more than three responses. and the total responses to each stimulus tor each subgroup is shown in Table 2. Due to

Table 2

Number of Responses to Each Stimulus, $\chi^{2}$ Tests, and Number of Ss Showing a Particular or No Stimulus Preference (n.p.)

\begin{tabular}{|c|c|c|c|c|c|c|c|c|c|c|}
\hline \multirow[b]{2}{*}{ Drive Group } & \multirow{2}{*}{$\begin{array}{l}1 \cdots \text { Pair } \\
\text { Stimuli }\end{array}$} & \multicolumn{3}{|c|}{ a } & \multicolumn{3}{|c|}{$b$} & \multicolumn{3}{|c|}{$\stackrel{c}{c}$} \\
\hline & & $G$ & Gwe & $x^{2}$ & B & $B W C$ & $x^{2}$ & G & $B W C$ & $x^{2}$ \\
\hline \multicolumn{11}{|l|}{ Experimental } \\
\hline \multirow{2}{*}{\multicolumn{2}{|c|}{$\begin{array}{l}\text { HI Responses } \\
\text { Preference (n.p.) }\end{array}$}} & 12 & 18 & 1.20 & 2 & 28 & $22.54 * *$ & 18 & 12 & 1.20 \\
\hline & & 1 & 2 & $(2)$ & - & 5 & $(-)$ & 2 & - & (3) \\
\hline \multirow{2}{*}{\multicolumn{2}{|c|}{$M O D$}} & 7 & 23 & $8.53^{* *}$ & 1 & 29 & $26.13^{* *}$ & 7 & 23 & $8.53^{* *}$ \\
\hline & & 1 & 4 & $(--)$ & - & 5 & $(-)$ & - & 4 & (1) \\
\hline \multirow{2}{*}{\multicolumn{2}{|c|}{$\mathrm{HI} / \mathrm{MOD}$}} & 9 & 21 & $4.80^{\circ}$ & 1 & 29 & $26.13^{* *}$ & 18 & 12 & 1.20 \\
\hline & & - & 3 & $: 2)$ & - & 5 & $(-)$ & 3 & 1 & (1) \\
\hline \multirow{2}{*}{\multicolumn{2}{|c|}{$\mathrm{MOD} / \mathrm{HI}$}} & 13 & 17 & 0.53 & 2 & 28 & $22.54 * *$ & 8 & 22 & $6.53 * *$ \\
\hline & & 2 & 3 & $(-)$ & - & 5 & $(-)$ & - & 5 & $(-)$ \\
\hline \multicolumn{11}{|l|}{ Control } \\
\hline \multirow{2}{*}{\multicolumn{2}{|c|}{$\mathrm{HI}$}} & 13 & 17 & 0.53 & 5 & 25 & $13.33^{* *}$ & 18 & 12 & 1.20 n.s. \\
\hline & & 1 & 3 & (1) & 1 & 4 & $(-)$ & 1 & 3 & (1) \\
\hline \multirow{2}{*}{\multicolumn{2}{|c|}{ MOD }} & 13 & 17 & 0.53 & 1 & 29 & $26.13 * *$ & 11 & 19 & $2.13 \mathrm{ns.s.}$ \\
\hline & & 1 & 3 & (1) & - & 5 & $(-)$ & 1 & 3 & (1) \\
\hline
\end{tabular}
ignifesults. In Test Pair b, all subgroups

Ss preferred the Bwc door to the $G$ door, however. Therefore, the original G+ vs Bhabit strength was weaker for MOD Ss than for $\mathrm{HI}$ Ss and thus more easily disrupted or changed by the inclusion of a redundant cue.

Results for Test Pair a did not correspond with those for Test Pair c. It is possible that each type of pair represented a very different kind of task. The redundant cue could have been attended to $B$ door. In Test c, only MOD/MOD and MOD/HI showed any significant preference, in this case to the Bwc over the $\mathrm{G}$ door. $\mathrm{HI} / \mathrm{HI}$ and $\mathrm{HI} / \mathrm{MOD} \mathrm{Ss}$, contrary to predictions. failed to show any preference, let alone a preference for the $G$ stimulus door. The lack of preference by MOD as well as HI control $S s$ points to the possibility that MOD experimental Ss reacted to the cue stimulus as a positive reinforcing cue rather than as a novel stimulus.

\section{CONCLUSION AND DISCUSSION}

The present study suggests that habit strength as well as performance in a discrimination task is affected by drive level. Predictions that drive level during original discrimination learning would be shown in presentations of Test Pair a and Test Pair c were only validated in the latter. In Test Pair a. a drive performance

effect was shown, while in Test Pair $\mathrm{c}$ a possible differentiation of habit strength during original discrimination learning was found. Although experimental Ss trained under $\mathrm{HI}$ deprivation conditions responded more to the $\mathrm{G}$ door in the testing situation than Ss trained under MOD deprivation conditions. HI experimental Ss did not show the expected preference for the $G$ door to the Bwc door. MOD experimental

either as an extra dimensional stimulus or as a stimulus within the already learned brightness dimension. The latter possibility is suggested by the overwhelming preference by all subgroups for the Bwc rather than B stimulus during Test Pair $b$ presentations. Test Pair a, Gwc vs G stimuli may be considered a within-dimension or transposition task, while Test Pair c may be considered an extradimensional shift task. The present study suggests that drive level may show only performance effects, if any, through within-dimensional shifts and learning effects of discrimination learning through extradimensional shifts of stimuli. The main reason for lack of learning differences due to drive level in the Eisman et al study (1956) may be due to the exclusive use of within-dimensional shifts in the stimuli, i.e., reversal learning.

\section{REFERENCES}

BRUNER, J. S.. MATTER, J., \& PAPANEK, M. L. Breadth of learning as function of drive and mechanization. Psychological Review, 1955, 62, 1-10.

CHAPMAN, R. M., \& LEVY, N. Hunger drive and reinforcing effect of novel stimuli. Journal of Comparative \& Physiological Psychology, 1957, 50, 233-238.

COHEN, J. S., \& STETTNER, L. J. Effect of deprivation level on exploratory behavior in the albino rat. Journal of Comparative \& Physiological Psychology, 1968, 66, 514-517. COHEN, J. S., STETTNER, L. J., \& MICHAEL, D. J. Effect of deprivation level on span of attention in a multi-dimension discrimination task. Psychonomic'Science, 1969, 15, 31-32.

EASTERBROOK, J. A. The effect of emotion on cue utilization and the organization of behavior. Psychological Review, 1959, 66, 183-201.

EISMAN, E., ASIMON, A., \& MALTZMAN, I. Habit strength as a function of drive in a brightness discrimination problem. Journal of Experimental Psychology, 1956, 51, 58-64.

FELLOWS, B. Chance stimulus sequences for discrimination tasks. Psychological Bulletin, 1967, 67, 87-92.

SUTHERLAND, N. S., \& HOLGATE, V. Two cue discrimination learning in rats. Journal of Comparative \& Physiological Psychology, 1966.61, 198-207.

TOLMAN, E. C. Cognitive maps in rats and men. Psychological Review. 1948. 55, 189-208. 